\title{
Análisis De La Problemática Y Enfoque Entorno A La Elaboración De Envases Biodegradables A Partir De Subproductos Del Maíz
}

\author{
Analysis of the problem and approach around the production of biodegradable packaging from \\ maize by-products
}

\author{
Mera Intriago Yerlit Jamilex ${ }^{1}$ \\ Universidad Técnica Estatal de Quevedo \\ Rendón Pozo Ariana Valeria ${ }^{2}$ \\ Universidad Técnica Estatal de Quevedo \\ Azucena Elizabeth Bernal Gutiérrez ${ }^{3}$ * \\ Universidad Técnica Estatal de Quevedo \\ Leonardo Arturo Baque Mite ${ }^{*}$ (ID \\ Universidad Técnica Estatal de Quevedo
}

Fecha recepción: 15 de junio de 2020

Fecha aceptación: 16 de julio de 2020

(1) 2020 Universidad de Cordoba. Este es un artículo de acceso abierto distribuido bajo los términos de la licencia Creative Commons Attribution License, que permite el uso ilimitado, distribución y reproducción en cualquier medio, siempre que el autor original y la fuente se acreditan.

1 Estudiante De La Universidad Técnica Estatal de Quevedo, Facultad de Ciencias de Ia Ingeniería, Quevedo, Ecuador, yerlit.mera2013@uteq.edu.ec https://orcid.org/0000-0002-3873-3571

2 en Ciencias, Especialización Químico Biológicas, Estudiante, Facultad de Ciencias de la Ingeniería, Universidad Técnica Estatal de Quevedo, Quevedo, Ecuador, ariana.rendon2016@uteq.edu.ec https://orcid.org/0000-0002-5532-7794 Ecuador, abernal@uteq.edu.ec https://orcid.org/0000-0003-2917-6408

4 Magister, Facultad de Ciencias de la Ingeniería, Universidad Técnica Estatal de Quevedo, Quevedo, Ecuador, Ibaque@uteq.edu.ec https://orcid.org/0000-0003-3079-4617 


\section{RESUMEN}

El plástico se ha convertido en un material muy demandado en la actualidad por sus características físico-químicas, lo cual permite ser muy aplicable en diferentes productos para diversos usos. Sin embargo, su producción masiva y mala gestión de residuos provocan un grave problema medioambiental de difícil solución, National Geographic, registró una producción mundial de 407 millones de toneladas de plástico hasta el 2015, los cuales tardan más de 1000 años en degradarse, debido a que se producen a partir del procesamiento de combustibles fósiles. Por tal razón, se busca la no dependencia de dicho plástico empleando nuevas alternativas como los plásticos biodegradables, los cuales presentan alta degradabilidad ante los compuestos orgánicos presentes en el medio ambiente. El almidón es una de las principales materias primas consideradas para tal fin, debido a sus características beneficiarias en comparación al petróleo. Para su extracción existen diferentes métodos, como son la molienda seca de forma artesanal, la húmeda usada por grandes industrias para refinar y mejorar la obtención del almidón y la nixtamalización que trabaja de la mano con el método de molienda húmeda. En Ecuador existe gran actividad agrícola, por ende, es de interés el aprovechamiento de subproductos y residuos de la agroindustria tales como los granos de rechazo de maíz, el elote y la fécula de maíz, consideradas como fuentes alternativas no convencionales para la producción de almidón en la elaboración de bioplásticos compitiendo con el plástico tradicional debido a que cuentan con apropiadas características y degradación del $89,40 \%$ en 90 días.

\section{ABSTRACT}

Plastic has become a very demanded material nowadays due to its physicalchemical characteristics, which allows it to be very applicable in different products for several uses. However, its massive production and poor waste management cause a serious environmental problem of difficult solution, National Geographic, recorded a global production of 407 million tons of plastic until 2015, which take more than 1000 years to degrade, because they are produced from the processing of fossil fuels. For this reason, we seek to avoid dependence on this plastic by using new alternatives such as biodegradable plastics, which are highly degradable to the organic compounds present in the environment. Starch is one of the main raw materials considered for such fin, due to its beneficial characteristics compared to oil. For its extraction there are different methods, such as dry milling by hand, wet milling used by large industries for refine and improve the obtaining of starch and nixtamalization that works hand in hand with the method of wet milling. In Ecuador, there is a great deal of agricultural activity, therefore, it is of interest to take advantage of by-products and residues from agroindustry such as corn rejection grains, corn and cornstarch, considered as alternative non-conventional sources for the production of starch in the elaboration of bioplastics competing with traditional plastic because they have appropriate characteristics and $89.40 \%$ degradation in 90 days. 
PALABRAS CLAVE: biopolímero, bioplásticos, almidón, degradabilidad
KEYWORDS: biopolymer, bioplastics, starch, degradability

\section{INTRODUCCIÓN}

La producción masiva de materiales plásticos y la mala gestión de sus residuos hacen que en la actualidad se haya generado un grave problema medioambiental de difícil solución (Prieto, 2020). Dado que la gran mayoría de los plásticos se producen a partir del procesamiento de los combustibles fósiles, se ha cuestionado su contribución al agotamiento de estos recursos y a los efectos ambientales de su extracción (Pérez, 2010).

Hoy en día, la contaminación de aguas con deshechos de materiales plásticos y sus fragmentos es reconocido como uno de los mayores problemas de calidad en los sistemas de aguas dulces y marinas (A. Koelmans, 2015), y ha sido documentada por varios estudios recientes dedicados a la identificación de fuentes, la abundancia y el impacto ambiental de los deshechos de plásticos (Andrady, 2011), (Hammer, 2012), (Browne, 2015), (François Galgani, 2015), (Thompson, 2015).

En el año 2005, PNUMA (Programa de Naciones Unidas para el Medio Ambiente) asegura que por kilómetro cuadrado se encontraban unas trece mil partículas plásticas flotando en el fondo del mar; otra de las preocupaciones es la cantidad de fragmentos diminutos de plástico incluyendo los PET que flotan en el Pacífico, los cuales han aumentado unas
100 veces en las últimas cuatro décadas, afirman científicos de la Scripps Institution of Oceanography, quienes culminaron una investigación sobre el aumento de estos residuos y sus efectos en los ecosistemas marinos. Además, afirmaron los oceanógrafos, que esta acumulación de basura plástica afecta a la fauna de múltiples maneras (Goldstein, 2012).

De acuerdo a la investigación realizada por la universidad politécnica de Madrid en conjunto a la empresa Ecoembes, aunque la capacidad productiva mundial de biopolímeros estimada en 2007 era de unas $300.000 \mathrm{t}$, lo que no supone ni el $1 \%$ del consumo total mundial de plástico, se esperó un alto crecimiento del sector de envases fabricados con bioplásticos: entre 700.000 y 1 millón de toneladas para el 2011, según escenarios de crecimiento del precio del petróleo, entre 3 y 5 millones de toneladas para el 2020 , siempre y cuando el precio del barril de petróleo esté por encima de los 200\$ en ese año (Medioambiente, 2009).

Según el Inec, en Ecuador una de las posibles soluciones adoptadas a esta problemática ha sido el reciclaje mediante la clasificación de los residuos sólidos. Un $37,1 \%$ de gobiernos municipales cuenta con procesos de separación en la fuente; es decir, diferencian los materiales orgánicos e inorgánicos (cartón, papel, plástico, vidrio, madera, metal, chatarra, caucho, textil, focos, pilas y desechos sanitarios no 
peligrosos, entre otros). Teniendo un porcentaje del $47,47 \%$ de que en los hogares realizan la clasificación de los residuos, siendo el plástico el principal residuo clasificado con el 32,98\% (Inec, 2017).

Es por esto que se busca la no dependencia de dicho plástico, empleando nuevas soluciones como los plásticos biodegradables (Galvez, 2016), que entre sus ventajas presenta su degradabilidad en compuestos tales como: agua, dióxido de carbono y humus (Adriana Avellán, 2019), además según la investigación de González (2009) los biopolímeros o bioplásticos se caracterizan en degradarse en un período de 45 o 90 días aproximadamente después de ser desechados, y también porque pueden ser empleados en la elaboración de empaques, vasos, películas plásticas y papel de revestimiento, en la actualidad se producen mayoritariamente a partir de residuos industriales y urbanos con alta carga de materia orgánica, aunque también pueden producirse a partir de gases como el CO o el CO2 (Prieto, 2020).Los bioplásticos constituyen la oportunidad estratégica para la exigencia medio ambiental gestada durante estos últimos años, debida a la acumulación de materiales sintéticos (Rodríguez, 2012), la perspectiva económica de los biopolímeros, polímeros obtenidos de recursos renovables a partir de los cuáles se pueden formular materiales, demuestra un mercado actual de aproximadamente $110000 \mathrm{t}$ anuales, con una elevada tasa de crecimiento una menor dependencia de los combustibles fósiles, una alta aceptación entre los consumidores (comprobadas con estudios de mercados) y sobre todo una elevada inversión en investigación (Chang, 2016).

Los bioplásticos son compuestos de alto peso molecular elaborados a partir de fuentes naturales, tales como cultivos de poliésteres microbianos, de almidón, celulosa, entre otros. El almidón ha sido una de las principales materias primas consideradas para tal fin, debido a su alta disponibilidad, bajo costo, carácter renovable, biodegradabilidad $y$ competitividad económicamente en relación al petróleo (Adriana Avellán, 2019).

El maíz es uno de los cereales que más se produce y consume a nivel mundial. Se utiliza principalmente para fines alimentarios, además de la producción de biocarburantes, para extraer almidón, aceite y otras sustancias destinadas a usos industriales. El Departamento de Agricultura de Estados Unidos (USDA) estima que la Producción Mundial de Maíz 2020/2021 sería de 1,186.86 millones de toneladas. La Producción de Maíz del 2019 fue de 1,114.75 millones de toneladas. Los $1,186.86$ millones de toneladas estimados para este año podrían significar un incremento de 72.10 millones de toneladas o $6.47 \%$ en la producción de maíz alrededor del mundo (Produccionagricolamundial, 2020).

Según los datos de Inec (2017), en Ecuador se produjeron cerca de 1,2 millones de toneladas de maíz en las más de 200000 hectáreas sembradas en el país, la provincia de Los Ríos es la mayor productora de $566.043 \mathrm{Tm}$ (Toneladas métricas) de maíz duro, Manabí 355.230 Tm, Guayas 315.390 Tm y Chimborazo junto a otras provincias productoras de maíz cosecha $199.443 \mathrm{Tm}$. La producción de biomasa residual que genera un cultivo de maíz de grano (cañas, hojas, chalas y olotes), fluctúa entre 20 a 35 toneladas por hectárea. El estudio publicado por la revista Agronomy Journal, descubrió que 
la eliminación de residuos procedentes del maíz y del trigo repercutía de manera nefasta en la calidad del suelo y el entorno. La eliminación de más del $50 \%$ de los residuos agrícolas puede tener consecuencias negativas para la estructura del suelo: reducir la secuestración de carbono orgánico del suelo, aumentar la erosión del agua y reducir el ciclo de nutrientes y la producción de cultivos, especialmente en suelos inclinados y erosionables (Clay, 2019). Es por esto que esta investigación propone analizar la problemática y enfoque entorno a la elaboración de envases biodegradables a partir de subproductos del maíz, identificar el impacto que tienen los bioplásticos en el entorno y los subproductos usados para la elaboración del mismo, determinar los métodos utilizados para la extracción de almidón a partir de subproductos de maíz, e investigar los diferentes procesos para la elaboración de bioplástico comparando los diferentes criterios de autores sobre características de dichos bioplásticos, para así explicar cuáles son los beneficios que estos proporcionan y porqué son una alternativa a los plásticos derivados de la industria petroquímica, resaltando su potencial, biodegradabilidad y su origen renovable, siendo una ventaja favorable que estos mayoritariamente en la actualidad se producen a partir de residuos industriales y urbanos mediante procesos biotecnológicos siguiendo los principios de la economía circular que permiten brindar un valor agregado a los desechos agroindustriales (Prieto, 2020).

\section{MARCO TEÓRICO.}

Esta investigación se basó en el análisis bibliográfico de diferentes autores pudiendo determinar que existen diversos tipos de materiales usados para la elaboración de bioplásticos. De acuerdo a la investigación realizada por Cristhian Almeida (2014), los diferentes centros de acopio se encargan de almacenar y secar el grano de maíz para su posterior comercialización a las industrias alimenticias, posteriormente el maíz no seleccionado para ser comercializado es desechado, quemado, botado o recirculados a las tierras como abono, menospreciando las demás utilizaciones que se le pueden dar a estos desechos (Almeida, 2014).

Por otro lado, García, García, Olaya, Rosas, \& Vignolo (2019) redactaron que para elaborar bioplástico se requirieron como materiales el almidón (48 g) que se pudieron obtener a partir de la fécula de maíz y se utilizaron como materiales complementarios; agua destilada $(150 \mathrm{ml})$, glicerina $(16 \mathrm{ml})$, vinagre $(21 \mathrm{ml})$, pegamento de arroz (16 g), gelatina colapez (20 g) y agua no potable, obteniendo un bioplástico con mayor resistencia de tracción, dureza, permeabilidad y resistencia a la perforación; mientras que Adriana, Vellán; Dayana, Díaz; Angie, Mendoza; María, Zambrano; Yuly, Zamora; Antonieta, Riera María (2019) especifican que el almidón de maíz (5.72 g) obtenido a partir del elote mediante el método de nixtamalización en conjunto con la mezcla con agua destilada $(17 \mathrm{ml})$, glicerina $(1.7 \mathrm{ml})$ y ácido acético (3ml) sirvieron como materiales para la obtención de bioplástico, y se tuvo como resultado un alto nivel de degradación con un porcentaje de $89,40 \%$ en 42 días; 
también Md. Ruhul Amin Mohammad Asaduzzaman Chowdhury (2019) narraron que los materiales necesarios para la obtención de bioplástico son: almidón de maíz comercial (50 g) a partir del grano de desecho (20\% de amilosa, $10 \%$ de humedad, tamaño medio de partícula $18 \mu \mathrm{m}), 25$ g. glicerol (6\%), 30 $\mathrm{ml}$ de vinagre blanco (8\%) y nanopartículas de dióxido de titanio (21 $\mathrm{nm}$ ), obteniendo un bioplástico compuesto con características de mejoras modificadas por la adición de dióxido de titanio, debido a que se obtiene un biopolímero más versátil y fácil de mezclar con otros materiales poliméricos.

Otro tipo de bioplástico a partir del almidón de maíz comercial (5g) se lo combina con materiales como: glicerol (2 $\mathrm{ml})$, agua desionizada $(30 \mathrm{ml}), 5 \%$ solución de ácido acético ( $2 \mathrm{ml})$, fibras naturales como algodón, yute, pelo y lana $(2 \%, \quad 4 \%, \quad 6 \%, \quad 8 \%, \quad 10 \%) ; \quad$ los biocompuestos sobre la base de almidón que son materiales renovables, son una alternativa potencial para los plásticos convencionales ya que presentaron mayor resistencia mecánica según la investigación de A.S., Jethoo (2019).

Así también Ibrahim, M.Z. Norashikin y M.Z. (2009), en su investigación explicaron que para realizar bioplástico utilizaron almidón de la cáscara de maíz, quitosano y aditivos como solución de hidróxido de sodio $(\mathrm{NaOH})$, agua destilada, ácido clorhídrico $(\mathrm{HCl})$, teniendo como resultados un bioplástico con alcance del $100 \%$ de degradación en 270 días.

Entre otros materiales para elaborar bioplásticos también estaba la celulosa del tallo de maíz, según Felicia Alodia Marie Rentoy, Dinah Ruth Angot, Jimdel Ryu Mabaquiao,Aris Larroder (2020), teniendo como resultado propiedades mecánicas débiles en comparación con otros plásticos comerciales como el celofán y menor densidad del bioplástico, esto pudiendo ser por las impurezas presente en la hoja y el tallo de maíz.

\section{METODOLOGÍA}

\subsection{Métodos identificados} para la extracción del almidón

En cuanto a la extracción del almidón, Carrasco y Molocho (2018) en su investigación extracción de almidón redactaron que la extracción del biopolímero puede realizarse a nivel artesanal y también a un nivel más tecnificado y a mayor y a menor escala, dependiendo de cada empresa. Existen diferentes métodos de extracción de almidón ya sea provenientes de maíz, trigo, yuca, papa o plátano. Los principales y más generales son: El método seco y el método húmedo. Estos métodos resultaron bastante simples para la extracción de almidón de yuca, papa o plátano y parecieron un poco más sencillos para la extracción de almidón a partir de los cereales y el maíz.

Según la FAO (1993) sin importar de donde provenga el almidón (maíz, trigo, yuca, papa o plátano), existen dos métodos usados para su extracción: la molienda seca y la molienda húmeda. Específicamente para el maíz, se emplea la molienda seca de forma artesanal y la húmeda es usada por grandes industrias para refinar y mejorar los productos obtenidos de esta planta, siendo el principal fin la obtención de almidón. 
Por lo tanto, comparando las redacciones realizadas por Carrasco, Molocho y la Fao se puede afirmar que se utilizan exclusivamente dos métodos específicos para la extracción de almidón a partir de recursos naturales.

La FAO (1993) indica que para la obtención de almidón a partir del maíz es necesario utilizar la parte del maíz en donde se encuentre abundantemente este polisacárido, por esta razón es muy común utilizar los granos del maíz ya que el almidón es el componente químico principal de este al que corresponde un 72 a 73 por ciento del peso del grano.

Según García, García, Olaya, Rosas, \& Vignolo (2019) Para la obtención de almidón a partir de granos de maíz se realiza el siguiente procedimiento: se receptan los granos del maíz, se hace una selección y lavado de los granos para luego proceder a colocarlos en la máquina de secado, posteriormente los granos secos son triturados y macerados en un periodo de 30-50 horas en una solución de SO2 al $0.1-0.2 \%$, se realiza una segunda trituración, un filtrado y una centrifugación para eliminar la proteína y realizar un segundo secado para obtener almidón puro y seco.

En la investigación realizada por la revista Colón, Ciencias, Tecnología y Negocios (2019), la extracción del almidón de maíz se lleva a cabo a través de seis etapas: maceración, molienda húmeda, filtración de la suspensión, sedimentación, lavado y por último el secado del almidón, a este método que va de la mano con la molienda húmeda se lo denomina método de Nixtamalización. El método de nixtamalización permite obtener un maíz molido con un $45 \%$ de humedad, cuando el maíz se muele pierde su estructura, la masa resultante de la molienda consiste en fragmentos de germen, residuos del pericarpio $y$ endospermo unidos por el almidón parcialmente gelatinizado, y por las proteínas y lípidos emulsificados que proporciona pegajosidad y adhesividad (Octavio Lopez, 2009).

Autores como Medina, Mackenzie y Bermúdez (1990) explican que por lo general a nivel industrial el almidón se obtiene por vía húmeda, en donde el maíz se macera en agua sulfurada $(\mathrm{pH} 4)$ a $50^{\circ} \mathrm{C}$ durante 35-45 horas, con lo cual se hidrata la proteína y simultáneamente se reducen los enlaces disulfuro lo que permite la liberación de los gránulos de almidón de la red proteica.

Z. Zhang, H. Feng,Y. $N$ i u, S. R. Eckhoff (2005) utilizaron para la extracción del almidón a partir de pienso de maíz, el método de S. R. Eckhoff que consiste en la utilización de ultrasonido y molienda húmeda. Las muestras de pienso de maíz contendrían un $46,4 \%$ de almidón total, un $8,93 \%$ de proteína y un $6,12 \%$ de aceite. La composición aproximada de las muestras estaría basada en el peso seco. Siendo separado en cinco muestras de $75 \mathrm{~g}$ (db), luego se empaparían en $600 \mathrm{~mL}$ de agua desionizada, y después de 20 minutos, cada muestra se trataría en una de las cinco condiciones diferentes; sólo ultrasonido, ultrasonido seguido de molienda fina, molienda fina seguida de ultrasonido, molienda fina solamente, y ningún tratamiento control.

\subsection{Métodos identificados para la elaboración de bioplástico}

En lo que respeta a la obtención de biopolímeros, según la investigación de 
García, García, Olaya, Rosas, \& Vignolo (2019) para la elaboración de bioplástico a partir del almidón de la fécula de maíz, se realiza el siguiente proceso; se mezclan los gránulos de almidón con toda la materia prima e insumos disueltos, se cocina a una temperatura de $80^{\circ}$, posteriormente se ingresa la mezcla a los extrusores para obtener el polímero deseado.

Mientras que Adriana, Vellán; Dayana, Díaz; Angie, Mendoza; María, Zambrano; Yuly, Zamora; Antonieta, Riera María (2019) en su investigación para la elaboración del bioplástico, se llevó a cabo mediante la metodología de nixtamalización, colocando en un recipiente $17 \mathrm{ml}$ de agua destilada junto con 5.72 g de almidón de maíz extraído, el cual se mezcla constantemente hasta homogeneizar y evitando que la temperatura no exceda los $70^{\circ} \mathrm{C}$. Posteriormente se agrega gota a gota 1.7 $\mathrm{ml}$ de glicerina con agitación constante, para después añadir $3 \mathrm{ml}$ de ácido acético al $3 \% \mathrm{v} / \mathrm{v}$ homogenizando hasta que se deje de percibir vapor en la mezcla. Finalizado este proceso, se forma la lámina sobre una placa de vidrio, exponiéndola al sol para su secado y luego se retire el bioplástico obtenido.

En cambio, de acuerdo a la investigación de Md. Ruhul Amin Mohammad Asaduzzaman Chowdhury (2019) para fabricar el bioplástico de almidón, se añade $50 \mathrm{~g}$ de almidón de maíz en un vaso precipitado junto con $300 \mathrm{ml}$ de agua destilada, luego se agrega al vaso precipitado $25 \mathrm{gm}$ de glicerol y $30 \mathrm{ml}$ de vinagre blanco. Sucesivamente se coloca el vaso precipitado sobre el "agitador magnético de placa caliente" y se agita la mezcla durante $5 \mathrm{~min}$. Durante el calentamiento, el agitador debe estar encendido. Este proceso debe ocurrir rápidamente y debe revolverse hasta que la mezcla espese. Una vez que la mezcla esté completamente lista, habrá que removerla varias veces y luego verter la mezcla en papel de aluminio o tinte. Dependiendo del grosor del plástico, el tiempo requerido puede ser menor o mayor durante el secado. Luego, el plástico se mantendrá en un lugar fresco y seco.

Mientras tanto en la investigación realizada por A.S., Jethoo, (2019) para la elaboración del bioplástico, usó una solución preparada por $5 \mathrm{~g}$ de almidón comercial, mezclándolo con $30 \mathrm{ml}$ de agua, $2 \mathrm{ml}$ de glicerol y $2 \mathrm{ml}$ de ácido acético, sucesivamente añadiendo una cantidad variable de diferentes tipos de fibras naturales $(2 \%, 4 \%, 6 \%, 8 \%$ y $10 \%)$ siendo esta solución calentada en una placa a $150^{\circ} \mathrm{C}$ durante 15 minutos con agitación continúa, usando una varilla de vidrio. Cuando la solución se volvió viscosa y se perdió su transparencia, la calefacción se detuvo. Después de que la solución se aclaró de nuevo, se retiró de la placa caliente y fue esparcida en una hoja de aluminio para secar a temperatura ambiente.

Por otro lado, otros autores como MIJ Ibrahim, S.M. Sapuan, E.S. Zainudin, M.YM Zuhri (2020) redactan que para obtener películas compuestas, se aisla almidón de maíz (CS) de gránulos de maíz fresco y se obtiene una composición de $24,64 \%$ de amilosa , amilopectina $74,36 \%$, $10,45 \%$ de humedad, $7,13 \%$ de lípidos y 0,62 de ceniza, de tal manera la fibra de cáscara de maíz se limpia, deshidrata, se muele y examina a través de un tamiz de malla de $300 \mu \mathrm{m}$, mientras que la fibra de palma azucarera se limpia a fondo y se convierte en polvo, el plastificante de fructosa es proporcionado por evergreen Sdn y sucesivamente las películas híbridas 
basadas en CS se prepararan mediante la técnica convencional de fundición en solución. Luego se añade $5 \mathrm{~g}$ de CS puro a una solución acuosa de agua destilada (100 ml), la cual es calentada por una mezcla término-magnética a $85 \pm 3{ }^{\circ} \mathrm{C}$ durante $25 \mathrm{~min}$ con agitación continua para permitir que el almidón se gelatinase. A continuación, se añade a la solución un plastificante de fructosa con una concentración del $25 \%$ (p/p de almidón en polvo) junto con $\mathrm{CHF}$ con una concentración del $8 \%(\mathrm{p} / \mathrm{p}$ de almidón en polvo). El FPS en varias cargas (0, 2, 4, 6, $8 \%$ ) de base de almidón seco se utiliza como agente hibridante. El proceso de calentamiento con agitación continua se mantiene durante 25 minutos más. Después de eso, la solución gelatinizada es descargada uniformemente en una placa térmica de Petri. La placa con la solución fundida se seca en un horno de circulación de aire durante $24 \mathrm{~h}$ a $45^{\circ} \mathrm{C}$, y las películas formadas son retiradas suavemente de las placas manteniéndose en condiciones ambientales durante diez días.

Para la elaboración de los envases biodegradables existen muchos métodos como: el método redactado por García, García, Olaya, Rosas, \& Vignolo (2019), en donde se mezclan los gránulos de almidón con toda la materia prima e insumos disueltos, realizando una cocción y sucesivamente una extrusión para obtener el polímero deseado. Así también, como en la investigación de Adriana, Vellán; Dayana, Díaz; Angie, Mendoza;
María, Zambrano; Yuly, Zamora; Antonieta, Riera María (2019), el cual el método usado para la obtención del bioplástico se lleva a cabo mediante nixtamalización, homogenización, cocción, agitación y secado. Mientras que autores como Md. Ruhul Amin Mohammad Asaduzzaman Chowdhury (2019) explican que, para obtener bioplástico mediante agitación rápida, se debe colocar la mezcla de los materiales en un agitador magnético de placa caliente y verter la mezcla en el material deseado, ya sea papel, aluminio o tinte para proceder al secado.

Otros autores como A.S., Jethoo, (2019) explican que para la obtención del biopolímero se realiza la mezcla de materiales, agitación en placa caliente y secado en papel aluminio a temperatura ambiente. Mientras tanto, MIJ Ibrahim, S.M. Sapuan, E.S. Zainudin, M.YM Zuhri (2020) manifiestan que para obtener películas compuestas, se utiliza la técnica convencional de fundición en solución. Los métodos mencionados anteriormente permiten obtener el biopolimero sin ser moldeado en forma de envase. Según la investigación de García, García, Olaya, Rosas, \& Vignolo (2019) determina un flujograma el cual lleva a la obtención de bandejas biodegradables (ver figura 1), especificando las operaciones unitarias que se deben realizar para obtener un producto perfectamente elaborado: 


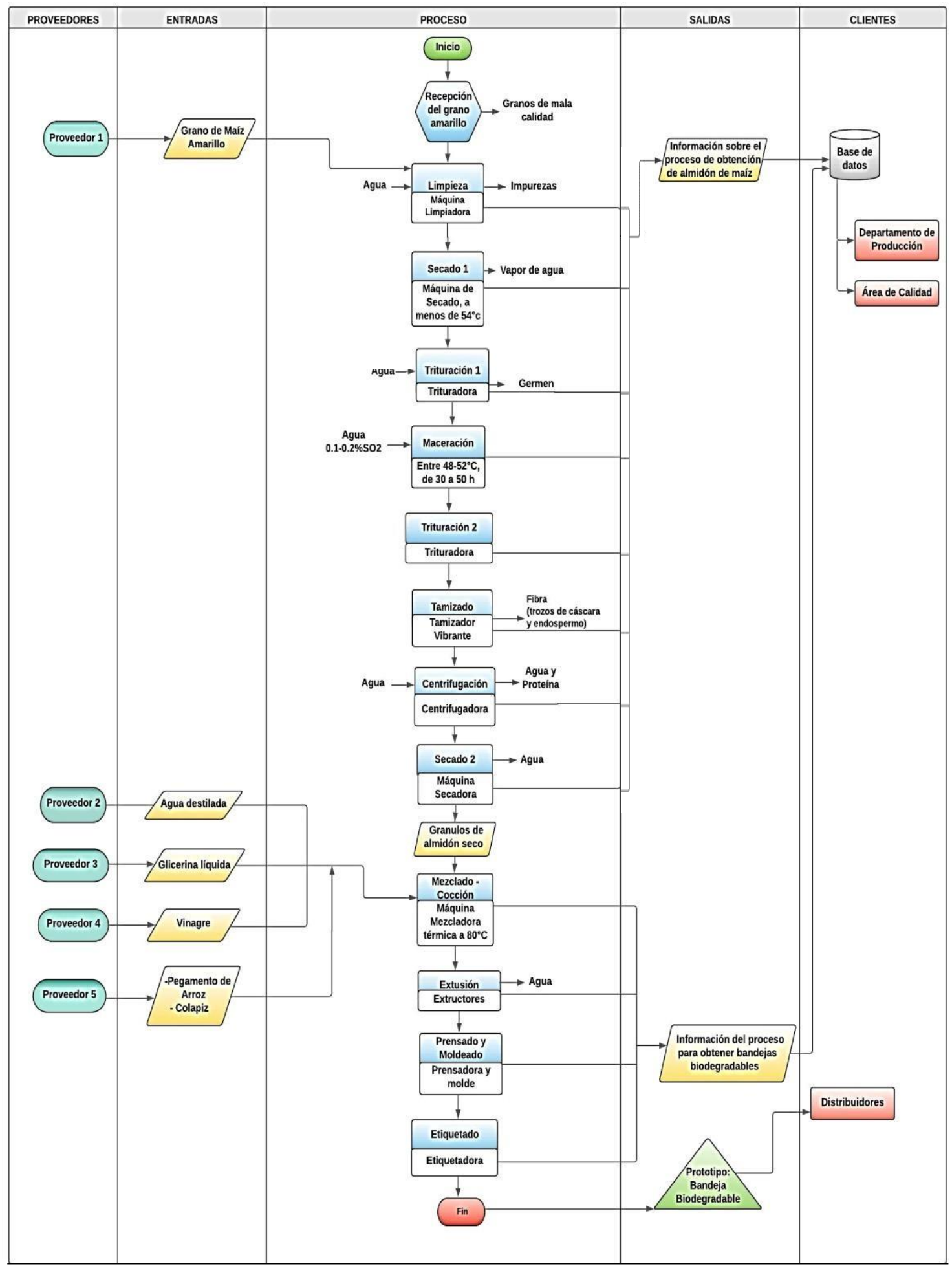

Figura 1. Flujograma para la obtención de envases biodegradables (García, García, Olaya, Rosas, \& Vignolo, 2019). 


\section{RESULTADOS}

Indagando las diferentes investigaciones sobre la obtención del almidón de maíz se observó que, muchos autores emplean la molienda seca de forma artesanal y la húmeda para la obtención de almidón de maíz, pudiendo afirmar que la molienda húmeda es el tipo de molienda más utilizada para obtener este polisacárido, entre los diferentes métodos utilizados. Los resultados de cada método comprobaron que en todas las investigaciones es importante el secado sin importar las operaciones unitarias previas a este último paso.

Según la investigación realizada por (Quiñonez, 2015) en su tesis “Obtención de un polímero biodegradable a partir de almidón de maíz", manifiesta que gracias a los recursos producidos por la tierra como el maíz, papa o yuca, entre otras, los cuales contienen almidón, al extraerlo este puede servir como base para producir un polímero biodegradable, con el cual se pueden fabricar bolsas para recolectar desperdicios orgánicos o empaques para productos varios (en forma de película, bandeja, etc.), demostrando que este polímero al ser compostado, puede descomponerse produciendo abono orgánico, el cual sirve como fertilizante de los suelos donde nuevamente producirán las plantas que contienen almidón. Por tal razón, es importante resaltar que el uso de los polímeros biodegradables tales como el almidón, pudieron ser una solución interesante a la problemática de los plásticos de petróleo, debido a su relativa abundancia y fácil biodegradabilidad (Quiñonez, 2015).

Acotando también, García, García, Olaya, Rosas, \& Vignolo (2019) explicaban que para medir las variables cualitativas estos realizaron las siguientes pruebas a las 5 muestras elaboradas en su investigación; Resistencia a la tracción, permeabilidad, dureza, perforación, temperatura y biodegradabilidad, en donde los mismos obtienen los siguientes resultados:

Tabla 1. Resistencia a la tracción (tensile strength)

\begin{tabular}{ccc}
\hline Muestra & Escala de fuerza & Tiempo \\
\hline $\mathbf{1}$ & Baja & 10 segundos \\
$\mathbf{2}$ & Baja & 5 segundos \\
$\mathbf{3}$ & Baja & 12 segundos \\
$\mathbf{4}$ & Baja & 18 segundos \\
$\mathbf{5}$ & Baja & 23 segundos \\
\hline
\end{tabular}

Fuente: (García, García, Olaya, Rosas, \& Vignolo, 2019) 
De tal manera, se pudo observar que en la prueba de resistencia a la tracción en la muestra 5, presentó mayor resistencia en la escala de fuerza, respecto a la permeabilidad, de las cuales todas las muestras realizadas lograron la impermeabilidad necesaria que necesita una bandeja, esto es comprobado vertiendo agua sobre las superficies planas de las bandejas, teniendo como resultado que en ninguna muestra pase agua sobre la superficie, al analizar la dureza de las muestras se obtiene como resultado que la bandeja que mostró mayor dureza y tuvo menor tiempo de secado fue la número 5 , debido a la consistencia que le otorga el colapiz con la goma de arroz como aditivos, los cuales influyeron positivamente en la forma de la bandeja, teniendo mayor fuerza y siendo la más lisa y con mejor forma, en cuanto a la prueba de perforación para hallar la fuerza máxima que puede resistir la bandeja, a esta se le midió el peso máximo que puede resistir hasta que se perfore la muestra 5 , siendo esta la que obtiene mejor resultado, de esta manera haciendo enfoque a la temperatura, estos determinaron la temperatura mínima y máxima, obteniendo como resultado que la bandeja 5 es la que tiene mejores características pudiendo resistir $-19^{\circ} \mathrm{C}$ como temperatura mínima y $120^{\circ} \mathrm{C}$ como temperatura máxima. De esta manera, ellos al analizar la biodegradabilidad observan que al medir las variables de demanda bioquímica de oxígeno a los 5 días (DBO5) y la demanda química de oxígeno, la bandeja de almidón de maíz es clasificada como materia orgánica moderadamente biodegradable (DQO). (García, García, Olaya, Rosas, \& Vignolo, 2019).
Por otro lado, en la investigación de Adriana, Vellán; Dayana, Díaz; Angie, Mendoza; María, Zambrano; Yuly, Zamora; Antonieta, Riera María (2019) en la elaboración de bioplásticos, se demostró que después de 42 días de experimentación estos procedieron a titular la cantidad de $\mathrm{Ba}(\mathrm{OH}) 2$ con el $\mathrm{HCl}$, notando un cambio en la coloración, sin embargo ellos evidenciaron la formación de un pequeño precipitado color blanco, el cual indica la formación de $\mathrm{BaCO} 3$ y comprueban con ello la degradación del bioplástico. Así mismo demostraron que al tamizar la muestra de tierra donde colocaron inicialmente el bioplástico, encontraron que aún quedaban sin degradar 0,53 g de este. Con este dato ellos calcularon el porcentaje de biodegradabilidad y, consideraron que el peso inicial del bioplástico colocado dentro del recipiente con tierra fue de $5,00 \mathrm{~g}$, obteniendo un $89,40 \%$ de biodegradación.

Un total de 20 muestras de bioplástico, reforzadas con fibras naturales como lana, algodón, pelo y yute, fueron preparadas y probadas para su fuerza mecánica en la investigación realizada por A.S., Jethoo, (2019), aquí se especificó que la resistencia de los bioplásticos reforzados con fibras también depende de la distribución de las fibras en la muestra, la propiedad de la resistencia a la tracción también se rigió por las propiedades de la fibra como, la fuerza mecánica, el espesor, etc.; siendo reforzados en los bioplásticos, obteniendo como resultado que las fibras de yute tienen la mayor resistencia mecánica, pero las muestras hechas de fibras de yute mostraron menor resistencia a la tracción media. Esto pudiendo deberse a que las fibras de yute eran mucho más 
gruesas que otras fibras naturales, aunque el algodón, la lana y el pelo tenian baja resistencia mecánica, obteniendo que estas muestras tuvieron una resistencia a la tracción más alta que el yute. Esto siendo posible a que el algodón, la lana y el pelo, permiten una mezcla adecuada y una distribución uniforme de las fibras naturales dentro de las muestras. Por lo tanto, en esta investigación los bioplásticos reforzados con lana mostraron la mayor resistencia a la tracción entre todas las muestras debido a la misma razón. La resistencia a la tracción de los bioplásticos reforzados con fibra de lana mostraron el máximo promedio de tracción de $25.7592 \mathrm{Kg} / \mathrm{cm} 2$ en comparación con los bioplásticos reforzados con algodón, pelo y fibras de yute. En donde acotaron que esto pudiera deberse a la mezcla adecuada y a la distribución uniforme de las fibras naturales dentro de las muestras de bioplástico.

En la investigación de MIJ Ibrahim, S.M. Sapuan, E.S. Zainudin, M.YM Zuhri (2020) para analizar películas compuestas examinaron las siguientes variables: densidad, contenido de humedad, absorción de agua, propiedades morfológicas, análisis ft-ir, análisis de difracción, propiedades termales, propiedades de tracción, propiedades de barrera al agua en donde los resultados de cada variable arrojaron lo siguiente: las películas biocompuestas híbridas obtenidas de almidón de maíz (CS) y residuos fibrosos tanto de cáscara de maíz (CHF) como de palma de azúcar (SPF) mediante el método de fundición en solución y deshidratación en los resultados experimentales y de caracterización mostraron una mejora significativa del rendimiento de la película compuesta tras el proceso de hibridación. La resistencia a la tracción y el módulo de Young de las películas híbridas aumentaron de 6,8 MPa a 19,05 MPa y de 61,15 $\mathrm{MPa}$ a 1133,47 MPa, respectivamente, ya que la película contiene un $6 \%$ de SPF, lo que la convierte en la carga de refuerzo más eficiente. Mientras que la permeabilidad al vapor de agua de los compuestos híbridos disminuyó en un $96,55 \%$ en comparación con la película CS nítida, lo que significó mejores propiedades de barrera al agua. El análisis de difracción reveló una notable mejora en el índice de cristalinidad después de la carga de la fibra del $15 \%$ al $27 \%$. La incorporación del SPF mejoró las propiedades físicas y causó una disminución insignificante de la estabilidad térmica de los compuestos producidos.

\section{CONCLUSIONES}

Identificando el impacto que tienen los bioplásticos en el entorno, se puede evidenciar por medio de varias investigaciones de diferentes autores que los bioplásticos o plásticos biodegradables son una de las soluciones factibles que se han propuesto para combatir la contaminación de nuestro entorno, y en gran parte de nuestros mares y ríos que son los más afectados por los plásticos de un solo uso, estos son recomendados por sus diversas ventajas, entre ellas su alta degradabilidad ante los compuestos orgánicos presentes en el medio ambiente, teniendo bioplásticos con periodos de degradabilidad entre 45 y 90 días, los cuales son empleados en diferentes productos como platos, vasos, utensilios de cocina, películas, empaques, etc. 
Determinando los subproductos para la realización de bioplásticos se demuestra que una de las ventajas para la elaboración de los mismos, es que su materia prima es a partir de fuentes naturales u orgánicas, las cuales son muy accesibles y de carácter renovable, por ende, los subproductos para la elaboración de bioplásticos mayormente usados son los subproductos de maíz, principalmente el almidón de maíz el cual ha sido una de las principales materias primas consideradas para tal fin, debido a su alta disponibilidad, bajo costo, carácter renovable, biodegradabilidad, propiedades fisicoquímicas y competitividad económicamente en relación al petróleo, así también otros subproductos del maíz como son la hoja de maíz, el grano de maíz de rechazo, la fécula de maíz, el elote de maíz, pienso de maíz y hasta el tallo de maíz.

Investigando los diferentes procesos para la elaboración de bioplásticos, se puede observar que existen diferentes métodos para la obtención del biopolímero, realizando este proceso mediante métodos como nixtamalización, homogenización, cocción, agitación $y$ secado, también por medio de agitación rápida, y también por fundición en solución, es sustancial resaltar que sin importar el método utilizado para la obtención de biopolímero, a nivel industrial y tecnificado, el diagrama de flujo propuesto por García, García, Olaya, Rosas, \& Vignolo (2019) permite la obtención de envases biodegradables mediante los procesos de prensado y moldeado del biopolímero.

Comparando los diferentes criterios de autores como (Quiñonez, 2015), (García, García, Olaya, Rosas, \& Vignolo, 2019), (Adriana Avellán, 2019), (A.S., 2019), (MIJ Ibrahim, 2020), se puede observar que cada uno tiene un criterio diferente en cuanto a la medición de variables que pueden calificar el bioplástico como apto o no apto para su posterior uso, esto quiere decir que cada método empleado requiere mayor enfoque y análisis en las variables propuestas por cada autor en cada investigación, probablemente esto se debe al uso de distintos materiales para la obtención del polímero, ya que cada material que se use como refuerzo para mejorar el bioplástico realizado con almidón, requiere de análisis específicos para las variables priorizadas en cada investigación.

\section{REFERENCIAS}

[1]. A. Koelmans, E. B. (2015). Nanoplastic in the aquatic environment," in Marine Anthropogenic Litter. Springer Open. doi:https://doi.org/10.1007/978-3319-16510-3_12

[2].A.S., J. (2019). Efecto del refuerzo de fibra sobre la resistencia a la tracción y la flexibilidad. de bioplástico a base de almidón de maíz. IOP Publishing, 1-6. doi:10.1088/1757899X/652/1/012035

[3].Adriana Avellán, D. D. (2019). Obtención de Bioplastico a partir de almidón de maíz. Revista Colón Ciencias, Tecnología y Negocios, 7(1), 1-11. Retrieved from https://revistas.up.ac.pa/index.ph $\mathrm{p} /$ revista_colon_ctn/article/view/ 1134/948

[4].Almeida, C. (2014). Diseño, Selección y construcción de un 
gasificador de desechos del maíz. Tesis de Grado, Universidad Estatal de Guayaquil, Guayaquil. Retrieved from

http: / / repositorio.ug.edu.ec/bitstr eam/redug/18751/1/Tesis\%20de\%2 0Cristhian\%20Almeida\%20Murillo.pd $f$

[5].Andrady, A. (2011). Microplastics in the marine enviroment Marine Pollution Bulletin (Vol. 62). North Carolina: Elsevier. Retrieved from https: / /www.sciencedirect.com/sc ience/article/pii/S0025326X110030 55

[6].Browne, M. A. (2015). Sources and pathways of microplastic to habitats. (M. B. Klages, Ed.) Springer, Cham. doi:https://doi.org/10.1007/978-3319-16510-3

[7].Chang, B. L. (2016). Desarrollo de una película biodegradable activa con liberación controlada de extracto de propóleo rojo cubano. Universidad de la Habana . La Habana: Editorial Universitaria. Retrieved from http://ebookcentral.proquest.com /lib/ueqsp/detail.action?docl

[8].Chowdhury, M. R. (2019). Caracterización y análisis de rendimiento de bioplásticos compuestos sintetizados mediante nanopartículas de dióxido de titanio con almidón de maíz. Sciencedirect, 12.

doi:https://doi.org/10.1016/j.heli yon.2019.e02009

[9].Clay, D. (2019). Desafíos del manejo de residuos de cultivos: un resumen de un número especial. Agronomy Journal.

[10]. FAO. (1993). Www.fao.org. Retrieved from http://www.fao.org/3/t0395s/T03 95S00.htm
[11]. Felicia Alodia Marie Rentoy, D. R. (2020). Development of Cellulose-based Bioplastic from Corn Stalks. Publisciences, 1 - 6. Retrieved from http://www.publiscience.org/wpcontent/uploads/2020/04/Develop ment-of-Cellulose-based-

Bioplastic-from-Corn-Stalks.pdf

[12]. François Galgani, G. H. (2015). Marine Anthropogenic Litter. (G. L. Bergmann M., Ed.) Springer, Cham. doi:https://doi.org/10.1007/978-3319-16510-3

[13]. Galvez, A. (2016). Elaboración de plásticos biodegradable a partir del almidón Extraído (Zea mays) (tesis de grado). Tesis de grado, Universidad de San Carlos de Guatemala , Guatemala. Retrieved from http://www.repositorio.usac.edu.g t/5102/

[14]. García, L. F., García, A. C., Olaya, P. C., Rosas, G. P., \& Vignolo, D. N. (2019). Diseño del proceso productivo de bandejas biodegradables a partir de fécula de maíz. Tesis de grado, Universidad de Piura, Piura. Retrieved from https: / / pirhua.udep.edu.pe/bitstr eam/handle/11042/4276/f15f56df2 c6ce4fa6b1beb82a733acaee5e1247 a3a2fc682d78384751f8c7955.pdf?se quence $=1 \&$ isAllowed $=y$

[15]. Goldstein, m. R. (2012). El aumento de los desechos microplástica. Biology letters, 5. doi: $10.1098 / \mathrm{rsbl} .2012 .0298$

[16]. González, U. (2009). El maíz y los productos de su industrialización. (D. F. México, Ed.) Máxico. Retrieved from https: / / biblio.uleam.edu.ec/cgibin/koha/opac- 
detail.pl?biblionumber $=5703$

[17]. Hammer, J. K. (2012). J. K. Hammer, "Plastics inthe marine environment: the dark side of a modern gift," . Reviews of Environmental Contamination and Toxicology, 44. doi:10.1007 / 9781-4614-3414-6_1

[18]. Ibrahim, M. N. (2009). The Potential of Natural Waste (Corn Husk) for Production of Environmental Friendly Biodegradable Film for Seedling. CiteSeerX, $176 \quad-180$. doi:10.1.1.193.3846

[19]. Inec. (2017). Instituto Nacional de Estadísticas y Censos. Retrieved from https: / /www.ecuadorencifras.gob. ec/documentos/web-

inec/Estadisticas_agropecuarias/es pac/espac_2017/Informe_Ejecutivo ESPAC_2017.pdf

[20]. Inec. (2017). Instituto Nacional de estadísticas y Censos . Retrieved from https: / /www.ecuadorencifras.gob. ec/documentos/webinec/Encuestas_Ambientales/Hogar es/Hogares_2017/DOC_TEC_MOD_A MBIENTAL_ENEMDU\%202017.pdf

[21]. Luis Carrasco, M. R. (2018). Extracción de almidón. Universidad Nacional Autónoma del Chota. El Chota: Calameo. Retrieved from https: / /es.calameo.com/read/005 193087c8fe3b2314cf

[22]. Medina, L. M., Mackenzie, J., \& Bermúdez, A. S. (1990). Obtención de almidón bajo en proteínas a partir de maíz desgerminado. Colombiana de química, 19, 1-8. Retrieved from https://revistas.unal.edu.co/index .php/rcolquim/article/view/15325 /16114

[23]. Medioambiente, C. d.
(2009). Proyecto de análisis de Bioplásticos. Universidad

Politécnica de Madrid. Universidad Politécnica de Madrid. Retrieved from

https: / /www.ecoembes.com/: https://www.ecoembes.com/sites /default/files/archivos_estudios_id i/proyecto_bioplasticos_-

_resumen_ejecutivo.pdf

[24]. MIJ Ibrahim, S. S. (2020). Journal of Materials Research and Technology. Sciencedirect, 9, 2002011.

doi:https://doi.org/10.1016/j.jmrt .2019.10.045

[25]. Octavio Lopez, F. G. (2009). La nixtamalización. Ciencias, 11. Retrieved from revistas.unam.mx

[26]. Pérez, A. V. (2010). Bioplásticos y plásticos degradables. Universidad Autónoma Metropolitana, Azcapotzalco . Retrieved from https: / /anipac.com/wpcontent/uploads/2018/09/bioplasti cos.pdf

[27]. Prieto, A. (2020, Agosto 1). Centro de Investigaciones Biológicas Margarita Salas. (S. E. Molecular, Ed.) doi:DOI: http://dx.doi.org/10.18567/sebbm div_ANC.2020.08.1

[28]. Produccionagricolamundial. (2020, Mayo). Retrieved from produccionagricolamundial.com: http: / /www.produccionagricolamu ndial.com/cultivos/maiz.aspx

[29]. Quiñonez, A. V. (2015). Obtención de un polímero biodegradable a partir de almidón de maíz. Tesis de grado, Escuela de Ingeniería Química, Santa Tecla. Retrieved from https: / /www.itca.edu.sv/wpcontent/themes/elaniinitca/docs/2015-Obtencion-de-un- 
polimero-biodegradable.pdf

[30]. Rodríguez, A. (2012). Biodegradabilidad de materiales bioplásticos. Ciencia y Tecnología de Alimentos (Vol. 22). D - Instituto de Investigaciones para la Industria Alimentaria. Retrieved from http://ebookcentral.proquest.com /

[31]. Thompson, R. C. (2015).
Marine Anthropogenic Litter. (G. L. Bergmann M., Ed.) Springer, Cham. doi:https: / /doi.org/10.1007/978-3319-16510-3 7

[32]. Z. Zhang, H. F. (2005). Starch Recovery from Degermed Corn Flour and Hominy Feed Using Power Ultrasound. Researchgate, 82, 3. doi:10.1094/CC-82-0447 